\title{
MALDI-TOF MS as a Label-Free Approach to Rapid Inhibitor Screening
}

\author{
Kenneth D. Greis, Songtao Zhou, Thomas M. Burt, Andrew N. Carr, \\ Elizabeth Dolan, Vijayasurian Easwaran, Artem Evdokimov, \\ Richard Kawamoto, Jeff Roesgen, and Gregory F. Davis \\ Procter and Gamble Pharmaceuticals, Mason, Ohio, USA
}

\begin{abstract}
Mass Spectrometry (MS) has been widely reported for measuring the conversion of substrates to products for enzyme assays. These measurements are typically performed by timeconsuming LC-MS to eliminate buffer salts that interfere with electrospray ionization MS. However, matrix-assisted laser desorption ionization, time-of-flight MS (MALDI-TOF MS) offers a label-free and direct readout of substrate and product, a fast sampling rate, and is tolerant of many buffer salts, reagents, and compounds that are typically found in enzyme reaction mixtures. In this report, a demonstration of how MALDI-TOF MS can be used to directly measure ratios of substrates and products to produce $\mathrm{IC}_{50}$ curves for rapid enzyme assays and compound screening is provided. Typical reproducibility parameters were $<7 \%$ RSD-a value comparable to ESI MS quantitative assays and well within the acceptable limits for screening assays. The speed of the MALDI readout is currently about $10 \mathrm{~s}$ per sample, thus allowing for over 7500 samples/day. From a simplicity standpoint, the enzymatic reaction mixtures are prepared by liquid handling robots, the reactions are stopped by addition of a 10 times volume of acidic matrix solution, and the samples are simultaneously transferred to MALDI target plate for analysis. Importantly, the ratios of substrate to product are of sufficient reproducibility to eliminate the need for internal standards and, thus, minimize the cost and increasing the speed of assay development. (J Am Soc Mass Spectrom 2006, 17, 815-822) (C) 2006 American Society for Mass Spectrometry
\end{abstract}

$\mathrm{M}$ ass spectrometry is the standard for tracelevel quantitation of compounds in complex mixtures [1-3]. In the pharmaceutical industry, this has led to the routine use of these techniques to monitor pharmacokinetics and bioavailability of drug compounds in biological matrices [1-3]. These measurements are typically performed by LC/ESI-MS/MS on triple quadrupole instruments to minimize the effects of other contaminating material in the mixtures [1-3]. Mass spectrometry techniques have also been widely reported for measuring the conversion of substrates to products for enzyme assays [4]. However, for many enzymes with peptide substrates, ESI-MS approaches have the added complication of dividing the substrate and product into multiple peaks due to charge-state distribution and thus affecting sensitivity and data extraction. Conversely, MALDI-TOF MS offers a fast sampling rate, primarily singly-charged peptides to simplify the data analysis, and a desorption method that is tolerant to many of the components present in enzyme reaction mixtures. This eliminates the need for

Published online April 17, 2006

Address reprint requests to Dr. K. D. Greis, Health Care Research Center, Procter and Gamble Pharmaceuticals, 8700 Mason-Montgomery Road, Mason, OH 45040, USA. E-mail: greis.kd@pg.com time-consuming prefractionation (i.e., LC separation) before analysis.

Early in the development of high end commercial MALDI-TOF instruments, Hsieh et al. [5] reported the use of a MALDI-TOF MS approach for automated, multi-enzyme compound screening. This approach took advantage of the speed and sensitivity of MALDITOF MS to provide a qualitative view of enzyme inhibition in the presence of multiple inhibitors. Furthermore, this group reported the ability to measure loss of activity (inhibition) for several enzymes simultaneously by monitoring for the appearance of three different enzyme products in one sample mixture. Like most multiplexing experiments, compound mixtures that showed activity could then be evaluated as individual components to determine which compound was responsible for the inhibitory activity. While this report demonstrated a qualitative measurement of enzyme inhibition, quantitative measurements are necessary for enzyme kinetics and the production of comparative $\mathrm{IC}_{50}$ values to rank inhibitor potency. Unfortunately, the reproducibility and quantitative capabilities of MALDI have always been questionable when compared to ESI methods. However, a growing body of literature has indicated that MALDI-TOF MS approaches can be effectively used for quantitative measurements [6-10]. These reports include direct quantitation of both small 
molecules [6] and larger peptides and biomolecules [7], relative quantitation of tagged peptides for proteomics studies [10], and quantitative measurements of enzyme reactions [11]. As is the case with ESI-MS/MS approaches, many of these methods rely on internal standards for the quantitation. For screening assays, a direct readout that requires no internal standard would be preferred because it would save the time and cost of synthesizing a suitable internal standard.

In this report, MALDI-TOF MS is evaluated as a tool to directly measure ratios of substrates and products to produce $\mathrm{IC}_{50}$ curves for rapid inhibitor compound screening. The general applicability of this approach for kinases (both ser/thr and tyr) and a series of related hydroxylases is assessed, and various parameters such as linearity and reproducibility are addressed. In addition, the application is extended to include both rank order of inhibitory potency and selectivity measurement for compounds against related enzymes. Furthermore, the advantages of this approach compared to traditional screening and other mass spectrometry methods is presented. Finally, the progress toward automating the overall process using commercially available products is discussed.

\section{Experimental}

\section{Materials}

$\alpha$-Cyano-4-hydroxycinnamic acid, $\alpha$-CHCA, (\#C2020) was from Sigma-Aldrich (St. Louis, MO). Ammonium monobasic phosphate (\#0776-01) was from J. T. Baker. Kemptide (\#22,593) and phosphorylated kemptide $(\# 60,528)$ were purchased from AnaSpec, Inc. (San Jose, CA). Neurogranin, the PKC $\alpha$ substrate peptide (\#V5611), was purchased from Promega (Madison, WI). All other substrate peptides (including the phosphorylated form of neurogranin) were synthesized by an in-house facility and purified to greater than $90 \%$ before use in the assays. Staurosporine (\#EI156-1000) was purchased from Biomol (Plymouth Meeting, PA). Protein Kinase C- $\alpha$ (PKC $\alpha, \# 12,946)$ and cAMP-dependent Protein Kinase, catalytic subunit (PKA, \#V5161) were purchased from Upstate Biotech (Charlottesville, VA) and Promega, respectively. PKC activation buffer (\#V226A) were purchased from Promega and used according to the supplier's instructions.

\section{Enzyme Assays and Sample Preparation}

All assay reactions were prepared as $10 \times 10$ arrays in the center section of a 384-well plate (Costar 3688, Corning Corporation, Corning, NY) using a Beckman Coulter Multimek (Fullerton, CA) equipped with 384well low volume transfer head. Compound dilutions and the peptide substrate containing the necessary cofactors (i.e., ATP, lipids, etc.) were prepared on separate plates using a Beckman Coulter Biomek 2000 workstation. The reactions were initiated, stopped, and transferred to MALDI target plates in the following order: (1) Five $\mu \mathrm{L}$ was transferred from the compound dilution plate to the assay plate containing $20 \mu \mathrm{L}$ enzyme and the appropriate buffer; (2) $25 \mu \mathrm{L}$ of the substrate peptide mixture (including cofactors) was transferred to the assay plate to start the reaction; (3) assay plates were incubated at RT for 30 to $60 \mathrm{~min}$, depending on the specific enzyme assay; (4) the reactions were stopped by transferring $5 \mu \mathrm{L}$ of the reaction mixture into $50 \mu \mathrm{L}$ of matrix solution $(5 \mathrm{mg} / \mathrm{mL}$ of $\alpha$-CHCA in $50 \%$ acetonitrile $/ \mathrm{H}_{2} \mathrm{O}, 0.1 \%$ TFA, $5 \mathrm{mM}$ ammonium monobasic phosphate; (5) two $\mu \mathrm{L}$ of the stopped reaction mixture was transferred onto a MALDI-TOF MS target plate, maintaining the original $10 \times 10$ array. The specific assay conditions for each enzyme reaction were as follows: PKA-enzyme activity was measured using 2.7 units/well PKA in $40 \mathrm{mM}$ Tris: $\mathrm{HCl}$, pH 7.5, containing $20 \mathrm{mM} \mathrm{MgCl} 2,0.1 \mathrm{mg} / \mathrm{mL}$ BSA, $50 \mu \mathrm{M}$ ATP, and $50 \mu \mathrm{M}$ kemptide substrate. Standard incubations for PKA were $30 \mathrm{~min}$ at RT. PKC $\alpha$-Enzyme activity was measured using $6 \mathrm{ng} /$ well $\mathrm{PKC} \alpha$ in Promega activation buffer, plus $0.1 \mathrm{mg} / \mathrm{mL}$ BSA, $50 \mu \mathrm{M}$ ATP, $200 \mu \mathrm{M}$ EDTA, $400 \mu \mathrm{M} \mathrm{CaCl}_{2}$, and 25 $\mu \mathrm{M}$ neurogranin substrate. Standard incubation conditions for PKC $\alpha$ were $30 \mathrm{~min}$ at RT.

\section{MALDI-TOF MS}

All spectra were collected on an Applied Biosystems (Foster City, CA) 4700 Proteomics Analyzer MALDITOF mass spectrometer equipped with a Nd:YAG laser (355 nm, $3 \mathrm{~ns}$ pulse width, $200 \mathrm{~Hz}$ repetition rate) in either positive or negative ion reflector mode as indicated. The system was operated with the 4700 Explorer software, version 2.0 or 3.0. Automated acquisition parameters were adjusted to capture and average only those individual spectra within defined success criteria. Specifically, signal intensities for the substrate peptide were set to a minimum threshold of 3000 counts and a maximum intensity of 65,000 counts. This ensured that neither null spectra nor saturated spectra were averaged into the final readout. Depending on the substrate peptide used, between 1000 and 2000 laser shots were averaged from a minimum of 25 random positions from each sample spot. Data were collected in triplicate from three successive days (unless otherwise noted) to capture the maximum variability related to preparation of the enzyme reactions, transfer of samples to MALDI target plates, data collection, and data extraction.

\section{Data Analysis}

The isotope cluster areas for the peptide substrate and product peaks were extracted into a Microsoft Excel worksheet from the $10 \times 10$ array of spectral data simultaneously using the automated analysis function provided within the 4700 Explore software. An algorithm within the 4700 Explorer analysis tools software defined the isotope cluster area based on the molecular 
weight and the general elemental composition of peptides. For further details about the background subtraction, peak thresholds, and determining the baseline for calculating the peak areas, contact Applied Biosystems, (Framingham, MA). The percent conversion ( $\%$ C) of substrate to product was calculated as the cluster area of the product $(\mathrm{P})$ divide by the sum of the cluster areas of the substrate $(S)$ and product multiplied by 100 as represented by eq 1 :

$$
\% \mathrm{C}=[\mathrm{P} /(\mathrm{P}+\mathrm{S})] \times 100
$$

Eq 1 is a measure of the ratio of product to substrate then solving for the $\% \mathrm{C}$. However, for concentrationdependent inhibition studies, inhibition was plotted as $\%$ maximal activity ( $\% \mathrm{MA}$ ) for the following reason. To measure inhibition of the enzyme activity, one must measure the degree to which that activity (\% C) was curtailed. Thus the concentration-dependant inhibition data were plotted as \% MA where the maximal activity is the $\% \mathrm{C}$ measured in control reactions (with no inhibitor) as represented in eq 2:

$$
\begin{aligned}
\% \mathrm{MA}= & (\% \mathrm{C} \text { with inhibitor } / \% \mathrm{C} \text { with } \\
& \text { no inhibitor }) \times 100
\end{aligned}
$$

Thus, the \% MA is the measurement of the degree of inhibition.

\section{Results and Discussion}

\section{Measuring Enzyme Activity by MALDI-TOF MS}

Mass spectrometry is ideally suited for any enzyme assay that results in a mass shift from substrate to product. For enzymes with peptide substrates, MALDITOF MS offers the advantage of speed, simplified spectra, and minimal sample handling. As a demonstration of the data obtained using this approach, kemptide substrate was incubated in the presence or absence of PKA and spotted onto a MALDI target plate as described in the Experimental section. The resulting mass increase of $80 \mathrm{Da}$ for the phosphorylation reaction corresponding to the expected enzyme activity, was readily detected (Figure 1). Similar results were obtained for other kinases and when incubating a hydroxylase enzyme with its peptide substrate to produce a mass increase of 16 Da corresponding to the hydroxylation reaction. While detection of the expected mass shift are indicative of enzyme activity, the relative quantitative relationship between the peak areas of the substrate and product as measured by MALDI-TOF MS remained to be determined.

For kinase reactions, one might expect dramatic changes in the ionization and detection efficiencies due to the increased acidity of the phosphorylated product when compared to the substrate peptide. One way to address this issue would be to use a deuterated internal standard for the phosphorylated product thus minimiz-

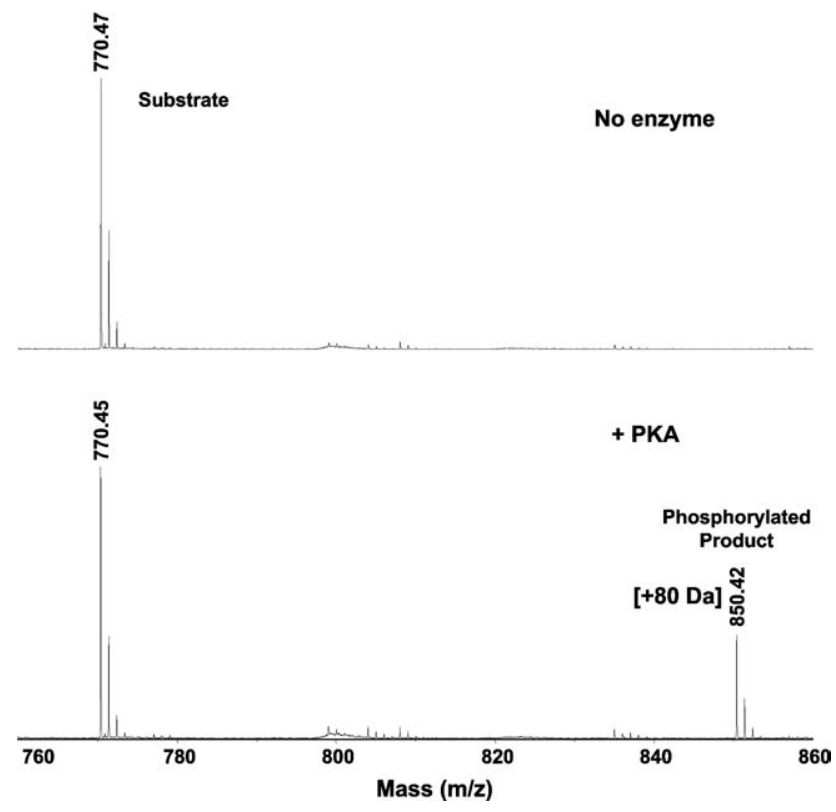

Figure 1. MALDI-TOF MS profiles for the enzyme reaction for PKA. Upper panel: Peptide substrate (kemptide) with buffers but no enzyme. Lower panel: Peptide substrates incubated with enzyme for $30 \mathrm{~min}$ resulting in the conversion of a portion of the substrate to the expected phosphorylated product. The position of the phosphorylation was confirmed by MS/MS fragmentation by TOF/TOF analysis (data not shown)

ing issues related to ionization efficiency differences. This has been the method of choice for quantitative MS for many years and has also recently been demonstrated by MALDI-TOF MS for small molecules $[6,8]$ and larger biomolecules [7]. However, to minimize the cost and maximize the speed of such direct quantitative experiments, we investigated whether simple ratios of substrate to product maintained a linear relationship that could then allow for accurate relative measurements to be made without internal standards. To investigate the linearity of the MS response, $50 \mu \mathrm{M}$ total peptide mixtures of kemptide and phosphorylated kemptide were spotted at known molar ratios onto the MALDI target plate. The ratios included 0, 2.5, 5, 10, 15, $20,25,30,35,40,45$, and 50\% product. Replicate samples of each ratio were prepared and analyzed on six separate days. Spectra were collected in negative ion reflector mode as described in the Experimental section. The percent product measured by MALDI-TOF MS was calculated as the isotope cluster area for the product (phosphorylated kemptide) divided by the sum of the cluster areas of the substrate (kemptide) plus the product multiplied by 100 . The average for the six replicates was plotted against the known molar ratios to produce the following best fit linear regression equation: $\mathrm{y}=$ $1.08 \mathrm{x}+3.6 ; \mathrm{r}^{\wedge} 2=0.998$, with \%RSD across all data points $\leq 8.0 \%$. The limit of detection was arbitrarily set to $2.5 \%$ product since this was the lowest value that we validated for relative quantitation using standard molar mixtures of substrate and product. The precision of measurement at the $2.5 \%$ product level were also below 
$8.0 \%$ RSD. The MS readout produced a slight overestimate for the phosphorylated product compared to the known molar ratios (slope $=1.08$ ). This was consistent with expectations since these data were collected in negative ion mode which would favor the ionization and detection of the phosphorylated product. Conversely, data collection in the positive ion mode produced a slight underestimate for the phosphorylated product $\left(\mathrm{y}=0.89 \mathrm{x}-1.01 ; \mathrm{r}^{\wedge} 2=0.992\right.$ with $\%$ RSD across all data points $\leq 7.8 \%$ ). Importantly however, in either case, the ratio of substrate to product produced a linear relationship, thus allowing for direct comparisons of relative enzyme activity for kinetics measurements. The negative ion mode was chosen in most cases as it minimized the level of $\mathrm{Na}^{+}, \mathrm{K}^{+}$, or $\mathrm{NH}_{4}{ }^{+}$adducts detected and, thus, simplified the data analysis.

Molar versus MS readout comparisons of substrate/ product combinations for additional enzymes (including, other serine/theronine and tyrosine kinases, and a hydroxylase enzyme) all yielded linear relationships with correlation coefficient $\left(\mathrm{r}^{\wedge} 2\right)$ values greater than 0.985 (data not shown), thus suggesting that this approach may be generally applicable to any enzyme assay utilizing a peptide substrates that produces a measurable mass shift. Furthermore, the effect of total peptide concentration (from 5 to $500 \mu \mathrm{M}$ ) was evaluated to determine whether higher molar concentrations of total peptide (substrate and product combine) would result in some deleterious or selective ionization of the substrates or products. Similar to the results presented above, the linear relationship was maintained across the peptide concentrations range with linear $\left(\mathrm{r}^{\wedge} 2\right)$ values all greater than 0.985 (data not shown). Taken together, these results suggest that simple ratios of substrates and products measured by MALDI-TOF MS are sufficient to measure relative enzyme activity and thus be applicable to screening the relative potencies of inhibitory compounds.

Another consideration when using substrate/product ratios to measure initial rate kinetics is that as the substrate begins to be depleted, the rate of conversion to product decreases rapidly. For ideal kinetic measurements, excess substrate would be used with direct quantitation of the product. However, by evaluating enzyme reactions at early stages, the initial rates can be maintained. Typically one targets the assay so that the product never exceeds $10 \%$ of the substrate concentration; however, when evaluating substrate/product ratios, the signal to noise begins to suffer at less than $20 \%$ conversions. Therefore, to determine the conditions at which the enzyme reaction maintained a close approximation of initial rate with sufficient signal to get an accurate readout, each enzyme reaction was evaluated through a time-course and at various enzyme concentrations to determine the linear range of the assay. The data from the time-course for PKA is shown in Figure 2. For the various classes of enzymes measured to date, the time and/or enzyme concentration could be adjusted to maintain a linear (initial) rate up to about $40 \%$

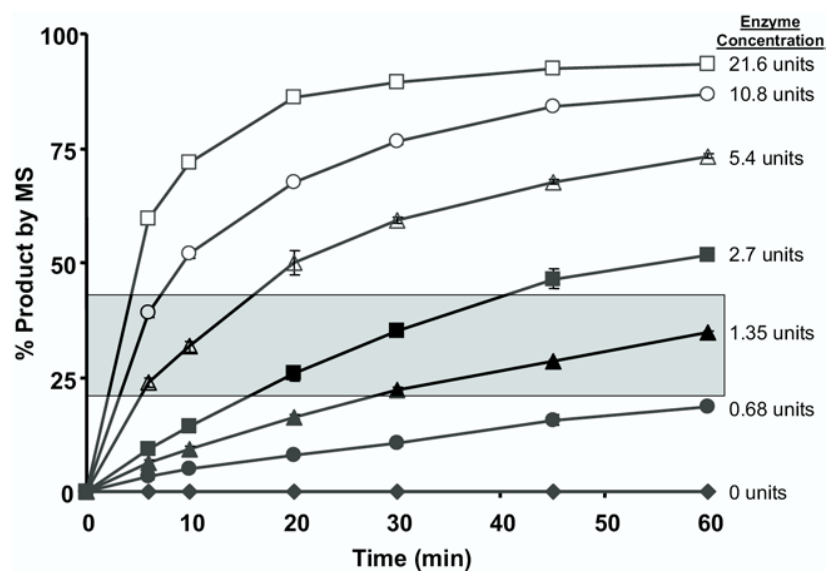

Figure 2. PKA enzyme titration and time-course at fixed substrate and ATP. To select an enzyme concentration and standard reaction time within an approximate linear range of the reaction (initial rate), a time-course at various PKA concentrations was evaluated. MALDI-TOF MS data were collected at the indicated time points. The data points represent the average and standard deviation of triplicate samples. The shaded area on the graph represents the target range (20-40\%) for measuring the \% product formed by MALDI-TOF MS. For this kinase, 2.7 units of enzyme for $30 \mathrm{~min}$ was chosen as the standard reaction conditions for further inhibitor screening.

conversion of substrate to product. At higher percentages of conversion, the velocity slowed down significantly due to depletion of substrate. Thus by setting up the control reactions to convert under $40 \%$ of substrate to product, a reasonable approximation of initial velocity could be maintained without need for saturating levels of substrate or isotopically-labeled internal standards.

\section{Accuracy and Precision}

For drug discovery and lead optimization, screening of compounds for inhibitory potency is central to the process. The process typically takes advantage of a known inhibitor as an introductory benchmark compound to develop and validate the assay and as an entry point for comparing potencies against new compounds. As a first step toward validating the utility of MALDI-TOF MS as a readout for these inhibitor assays, the reproducibility of the assay in a concentrationdependent inhibitor study was evaluated over 21 days (Figure 3). To capture the precision of these MS measurements (including the variability of sample preparation and analysis), the relative response from the control reactions were monitored over this same 21 day period. Experimentally, 10 control enzyme reactions were included on each $10 \times 10$ array to establish the baseline of control activity (maximal activity). These values were averaged for each plate and used to calculate the \% maximal activity as described in eq 2 in the Experimental section. Figure 3a shows the mean and standard deviation for 10 sets of control reactions across 21 days. The overall mean from 10 replicates of the PKA 

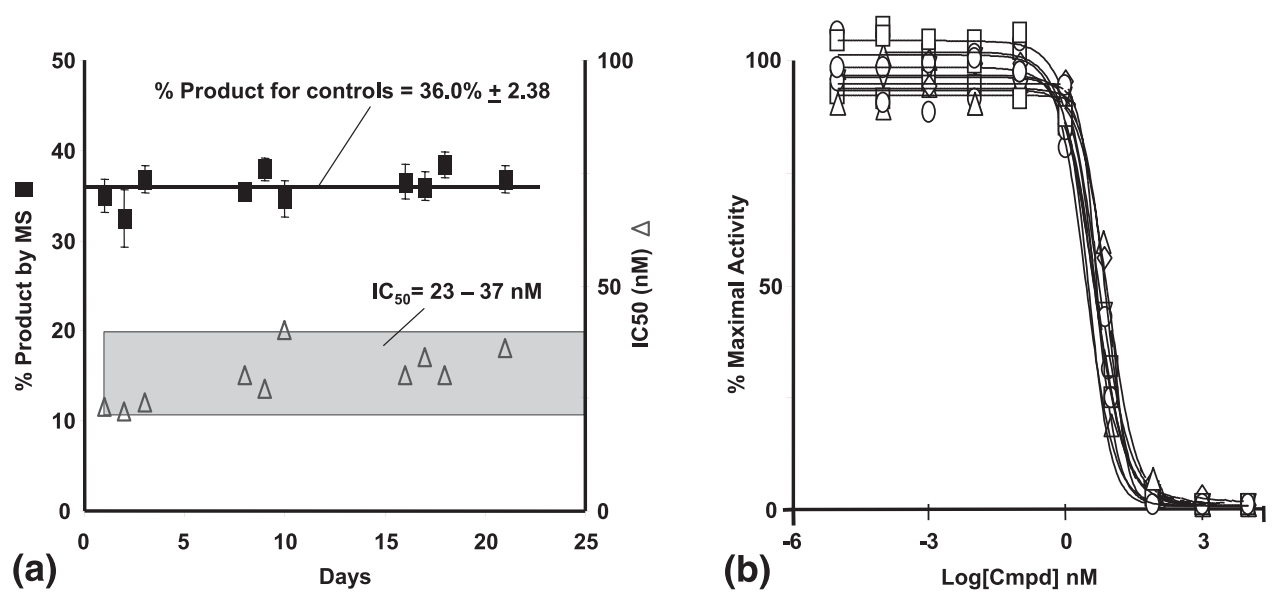

Figure 3. Stability of PKA inhibitory measurements over three weeks. Variability measurements and PKA inhibition curves with staurosporine, a benchmark kinase inhibitor, were generated over a period of 21 days. (a) Variability of the control reaction over 21 days is plotted in the upper portion the panel (left axis). For the days indicated, the \% product from 10 replicate control samples was plotted as a mean and standard deviation of 10 replicates on each day. Over the 21 days evaluated an overall mean and standard deviation of $36.0 \% \pm 2.38$ was determined resulting in a $\%$ RSD of just under $7 \%$. The calculated $\mathrm{IC}_{50}$ values for staurosporine were also plotted for each day in the lower portion of the panel (right axis). The values were derived from the individual $\mathrm{IC}_{50}$ curves shown in (b), a concentration-dependent inhibition of PKA with staurosporine measured on 10 days over a 21 day period to show the reproducibility of these measurements. The individual $\mathrm{IC}_{50}$ values are plotted in (a) with the shaded area showing an $\mathrm{IC}_{50}$ range of $23-37 \mathrm{nM}$ across the time period.

reaction on each of days (100 data points total) was $36.0 \% \pm 2.38$. This converts to a relative standard deviation of $<7 \%$ which is comparable to those produced for ESI-based quantitative measurements [1]. Thus the MS readout is demonstrated to have excellent precision even with all the variables associated with the sample preparations, data collection and data analysis. The ten $\mathrm{IC}_{50}$ inhibition curves for the benchmark compound (staurosporine) were plotted in panel 3B. The calculated $\mathrm{IC}_{50}$ values for PKA averaged about $29.6 \mathrm{nM}$ with a range from about 23 to $37 \mathrm{nM}$ (plotted individually in Figure 3a). Thus the relative ratios of substrate and product peaks are sufficient to generate the precision and accuracy needed for relative quantitation and screening assays.

\section{Rank Ordering of Inhibitor Potency}

Once the reproducibility and precision parameters were established, the ability to rapidly screen compounds to rank order inhibitor potency was investigated. Four different compound classes (staurosporine and three novel structures) were evaluated as inhibitors of PKA and plotted in Figure 4. The $\mathrm{IC}_{50}$ curves clearly demonstrated the ability to rank order compounds based on potency. In this case, staurosporine was the most potent inhibitor $\left(\mathrm{IC}_{50}=32 \mathrm{nM}\right)$, while compound $\mathrm{C}$ was the least potent $\left(\mathrm{IC}_{50}>100 \mu \mathrm{M}\right)$.

\section{Selectivity Measurements}

The potency of a compound against a selected target is only the first step in the drug discovery pipeline. Given that many enzymes exist as members of enzyme families with similar mechanism but often different functions, a potent inhibitor may only be useful as a drug if it can be demonstrated to be selective for the given target without interfering with other related enzymes. To that end, assays that allow for a comparison between potency of the inhibitor for the target enzyme and related enzymes are needed. To test this method for selectivity measurement, the relative potency of stauro-

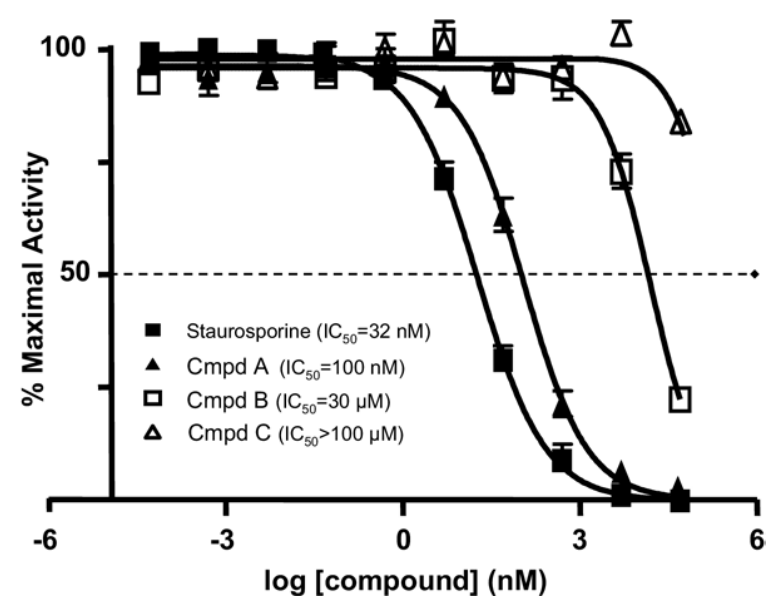

Figure 4. Rank ordering of PKA inhibitor potency. Enzyme assays were run with the indicated concentration of staurosporine and three novel compounds as described in the Experimental section. The percent maximal activity of substrate to product, as measured by peak cluster areas using MALDI-TOF MS, was plotted versus log compound concentration. The resulting comparative inhibition curves established relative $\mathrm{IC}_{50}$ values to produce the rank order of compound potency as inhibitors. 


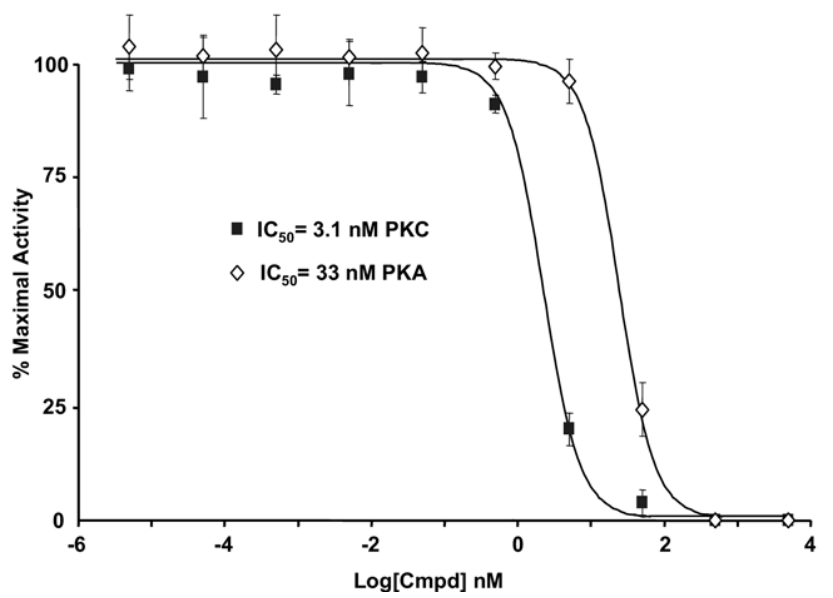

Figure 5. Evaluating selectivity of staurosporine for PKC $\alpha$ and PKA. Substrate peptides for PKA and PKC $\alpha$ were incubated under the established conditions for each enzyme to ensure linear responses at initial rate conditions. $0.5 \log$ dilutions of staurosporine were evaluated in a concentration-dependent inhibition study. The \% MA was plotted versus staurosporine concentration to produce the comparative $\mathrm{IC}_{50}$ curves.

sporine, a general kinase inhibitor, was compared for PKA and PKC $\alpha$. As plotted in Figure 5, staurosporine was 10 times more potent as an inhibitor for PKC $\alpha$ as compared to PKA. This is in agreement with literature reports on the relative inhibitory selectivity of staurosporine for these related enzymes [12, 13].

Similar selectivity assays have been developed for a series of Src family tyrosine kinases and a series of related hydroxylase enzymes. Like the PKA and PKC example shown here, the rank order and selectively of inhibitor potency could be readily determined using substrate/product ratios from the MALDI-TOF MS readout without need for internal standard or molar quantitation curves. Thus this approach has greatly improved the speed and cut down on the cost of our compound screening assays as compared to either traditional fluorescence or radiolabeled screening methods or LC-MS/MS based quantitative readout methods.

\section{Automation}

The current method is dependent on our ability to get the enzyme reaction mixtures onto the MALDI target plate. Given that the AB 4700 Proteomics Analyzer has a target plate with a $2 \times 2$ in. footprint, this was not directly amenable to simultaneous transfer of samples from a standard 96-well microtiter plate. Instead, to advance the automation, a $10 \times 10$ array format was developed in the center wells of 384-well plates, as this produced a footprint of the desired dimensions for direct sample transfer. Overall, the preparation of the enzyme/inhibitor assays was done via robotic liquid handling with the final quenching of the reaction by dilution into the acidic MALDI matrix mixture (see Experimental section for details). The automated spotting onto the target plate using the Multimek robot produced a uniform $10 \times 10$ array, ideally suited for the 4700 system. The $10 \times 10$ array coupled with an autoloader cassette on the source of the instrument allowed for the loading of up to 24 plates (2400 samples) for unattended data acquisition. Automated higher density spotting onto $2 \times 2$ inch target plates has been reported [5] and will be further investigated for this application. Alternatively, commercial MALDI-TOF MS instruments are available that accept microtiter platesized sample targets. These have been shown to accommodate both 384- and 1536-well formats, thus dramatically increasing the number of samples that could be loaded for unattended data collection.

\section{Why is an MS-Based Approach Desirable?}

As described in the Introduction, mass spectrometry is the industry standard for quantitative measurements [1-3]. Given that the quantitation of a substrate and/or product is the most direct measurement of an enzyme activity, coupling mass spectrometry to screening assays would then provide the most direct means of measuring inhibition of a given enzyme activity. Furthermore, in a screening setting, the other critical features of the assay readout are cost, speed, reproducibility, sensitivity, and automation. Issues related to false positive or false negative readings are also important considerations for a readout system. Importantly, the direct MS readout measurement of substrates and products eliminates the issues related to the false positives or negatives that can affect many of the traditional fluorescence, chemiluminescence, or radiological assays. Since these traditional assays often rely on a secondary (or coupled) reaction for the readout, they also increase the potential variability in the measurement. Furthermore, subsets of compounds from a screening library are likely to have inherent fluorescence properties, or may quench the fluorescence or chemiluminescence readout causing several false positive or false negative readouts from these assays. In addition, the use of unlabeled peptide substrates eliminates the need for costly fluorescent, chemiluminescent or radiolabeled reagents. Thus, MS methods offer a direct measurement of the substrate and product, avoid many of the false positive/negative issues related to traditional screening assays, and reduce the reagent costs.

MALDI-TOF MS approaches can offer significant advantages over LC-MS approaches based on the minimal sample preparation needed and the speed of the analysis. To date, nearly all the reports on the use of MS-based assays for compound screening rely on highly quantitative LC-MS approaches [14]. Some exceptions have included reports using porous siliconbased surfaces (DIOS) $[15,16]$ and tailored target surfaces to enhance the interaction of ligands with target proteins (SAMDI) [17]. In both of these approaches, inhibition curves, relative quantitation measurements and kinetic parameters were all demonstrated; however, these approaches require the preparation of spe- 
cialized surfaces or reagents not widely available to the research community. With the approach reported here, the ability to use commercial instrumentation and simple peptide substrates coupled with standard enzyme and inhibitor reactions for rapid compound screening is demonstrated. The direct measurement of substrate and product ratios provided the relative quantitation necessary to both rank order compounds for potency and to measure selectivity of compounds for related enzymes-all critical functions for screening assays.

\section{Future Directions}

This MALDI-TOF MS-based screening approach has now been used to measure enzyme activity and inhibition for nine different enzymes with peptide substrates ranging from 700 to $2800 \mathrm{Da}$. In all cases measured to date, the substrate/product ratios produced the linear relationships needed for relative quantitation. This suggests that these methods are amenable to a wide range of reactions with peptide substrates. Evaluation of a wider range of substrates and enzyme classes is needed to determine how broadly the approach can be used for screening. Given that numerous reports have emerged in the last few years demonstrating the quantitative capabilities for MALDI MS for small (non-peptide) molecules $[6,8,9,11]$, these results would imply that the screening methods reported here could be extended to enzymes with non-peptide substrates and products. The ability to find a suitable matrix for specific substrate product pairs and avoid interference from low molecular weight matrix clusters would likely represent the only challenge to making these approaches work. In initial tests with some small molecule substrates, we found that matrix interference could be minimized in the mass range between 200 and 700 Da by adding ammonium phosphate to the matrix [18] and by using a sufficient quantity of substrate such that it was maintained above the matrix background (data not shown). However, additional studies will be needed to determine the extent of such interference problems.

Even with the significant advantages that this approach offers, there are some limitations that are noteworthy. One limitation of MALDI-TOF MS is the need to have the analyte dry uniformly with the matrix material to assure good laser desorption and detection of the analytes. Thus, the ubiquitous use of DMSO for storage and dilution of compound libraries poses some challenges for cocrystallization with matrix. Similarly, other buffer components (salts, detergents, etc) can interfere with the formation of good matrix crystals. Fortunately, the sensitivity of most MALDI-TOF MS instruments (into the low femtomole range) is sufficient to allow the reaction mixtures to be diluted into matrix solution to minimize the effects of the buffer components. As described in the Experimental section, by using a 1:10 dilution of reaction mixtures into the matrix solution, interference from reaction mixture compo- nents did not affect the MS readout in any of the nine different screening assays we have evaluated to date.

\section{Conclusions}

We have demonstrated that MALDI-TOF MS offers a valid readout for compound screening assays. The speed, sensitivity, reduced cost, and reproducibility of this approach make it an ideal assay for dose-response curves for comparative $\mathrm{IC}_{50}$ measurements. Additional advances in laser pulse rates and sensitivity should improve the speed to the point of rivaling true high-throughput screening. Importantly, the direct nature of the MS measurement ensures the minimization of the false positive and false negative results that can plague other screening approaches. We have taken steps to validate the method and show a variability of $<7 \%$ RSD. These data confirmed that direct measurements of substrates and products ratios can be used to produce $\mathrm{IC}_{50}$ curves for rapid enzyme assays and compound screening without the need for labels or additional internal standards.

\section{Acknowledgments}

The authors thank Feng Wang and Michelle Tscheiner for providing high quality purified peptide substrates for many of the enzyme assays evaluated in this report.

\section{References}

1. Hoke, S. H.; II; Morand, K. L.; Greis, K. D.; Baker, T. R.; Harbol, K. L.; Dobson, R. L. M. Transformations in Pharmaceutical Research and Development Driven by Innovations in Multidimensional, Mass Spectrometry-Based Technologies. Int. J. Mass. Spectrom. 2001, 212, 135-196.

2. Hopfgartner, G.; Bourgogne, E. Quantitative High-Throughput Analysis of Drugs in Biological Matrices by Mass Spectrometry. Mass. Spectrom. Rev. 2003, 22, 195-214.

3. Taylor, P. J. Matrix Effects: The Achilles Heel of Quantitative HighPerformance Liquid Chromatography-Electrospray-Tandem Mass Spectrometry. Clinical. Biochem. 2005, 38, 328-334.

4. Liesener, A.; Karst, U. Monitoring Enzymatic Conversions by Mass Spectrometry: A Critical Review. Anal. Bioanal. Chem. 2005, 382, 14511464

5. Hsieh, F.; Keshishian, H.; Muir, C. Automated High Throughput Multiple Target Screening of Molecular Libraries by Microfluidics MALDI-TOF MS. J. Biomol. Screen. 1998, 3, 189-198.

6. Li, Y. L.; Gross, M. L. Ionic-Liquid Matrices for Quantitative Analysis by MALDI-TOF Mass Spectrometry. J. Am. Soc. Mass Spectrom. 2004, 15, 1833-1837.

7. Bucknall, M.; Fung, K. Y. C.; Duncan, M. W. Practical Quantitative Biomedical Applications of MALDI-TOF Mass Spectrometry. J. Am. Soc. Mass Spectrom. 2002, 13, 1015-1027.

8. Bungert, D.; Heinzle, E.; Tholey, A. Quantitative Matrix-Assisted Laser Desorption/Ionization Mass Sspectrometry for the Determination of Enzyme Activities. Anal. Biochem. 2004, 326, 167-175.

9. Sleno, L.; Volmer, D. A. Some Fundamental and Technical Aspects of the Quantitative Analysis of Pharmaceutical Drugs by Matrix-Assisted Laser Desorption/Ionization Mass Spectrometry. Rapid. Commun. Mass Spectrom. 2005, 19, 1928-1936.

10. Ross, P. L.; Huang, Y. N.; Marchese, J. N.; Williamson, B.; Parker, K.; Hattan, S.; Khainovski, N.; Pillai, S.; Dey, S.; Daniels, S.; Purkayastha, S.; Juhasz, P.; Martin, S.; Bartlet-Jones, M.; He, F.; Jacobson, A.; Pappin, D. J. Multiplexed Protein Quantitation in Saccharomyces cerevisiae Using Amine-Reactive Isobaric Tagging Reagents. Mol. Cell. Proteom. 2004, 12, 1154-1169.

11. Kang, M. J.; Tholey, A.; Heinzle, E. Application of Automated MatrixAssisted Laser Desorption/Ionization Time-of-Flight Mass Spectrometry for the Measurement of Enzyme Activities. Rapid. Commun. Mass Spectrom. 2001, 15, 1327-1333.

12. Tamaoki, T.; Nomoto, H.; Takahashi, I.; Kato, Y.; Morimoto, M.; Tomita, F. Staurosporine, a Potent Inhibitor of Phospholipid/Ca++ Dependent Protein Kinase. Biochem. Biophys. Res. Commun. 1986, 135, 397-402.

13. Matsumoto, H.; Sasaki, Y. Staurosporine, a Protein Kinase C Inhibitor Interferes with Proliferation of Arterial Smooth Muscle Cells. Biochem. Biophys. Res. Commun. 1989, 158, 105-109. 
14. de Boer, A. R.; Letzel, T.; van Elswijk, D. A.; Lingeman, H.; Niessen, W. M. A.; Irth, H. On-line Coupling of High-Performance Liquid Chromatography to a Continuous-Flow Enzyme Assay Based on Electrospray Ionization Mass Spectrometry. Anal. Chem. 2004, 76, 3155-3161.

15. Go, E. P.; Shen, Z.; Harris, K.; Siuzdak, G. Quantitative Analysis with Desorption/Ionization on Silicon Mass Spectrometry Using Electrospray Deposition. Anal. Chem. 2003, 75, 5475-5479.

16. Shen, Z.; Go, E. P.; Gamez, A.; Apon, J. V.; Fokin, V.; Greig, M.; Ventra, M.; Crowell, J. E.; Blixt, O.; Paulson, J. C.; Stevens, R. C.; Finn, M. G.;
Suizdak, G. A. Mass Spectrometry Plate Reader: Monitoring Enzyme Activity and Inhibition with a Desorption/Ionization on Silicon (DIOS) Platform. Chem. Biochem. 2004, 5, 921-927.

17. Min, D. H.; Tang, W.-J.; Mrksich, M. Chemical Screening by Mass Spectrometry to Identify Inhibitors of Anthrax Lethal Factor. Nat. Biotech. 2004, 6, 717-723.

18. Smirnov, I. P.; Zhu, X.; Taylor, T.; Huang, Y.; Ross, P.; Papayanopoulos, I. A.; Martin, S. A.; Pappin, D. J. Suppression of $\alpha$-Cyano-4-Hydroxycinnamic Acid Matrix Clusters and Reduction of Chemical Noise in MALDI-TOF Mass Spectrometry. Anal. Chem. 2004, 76, 2958-2965. 